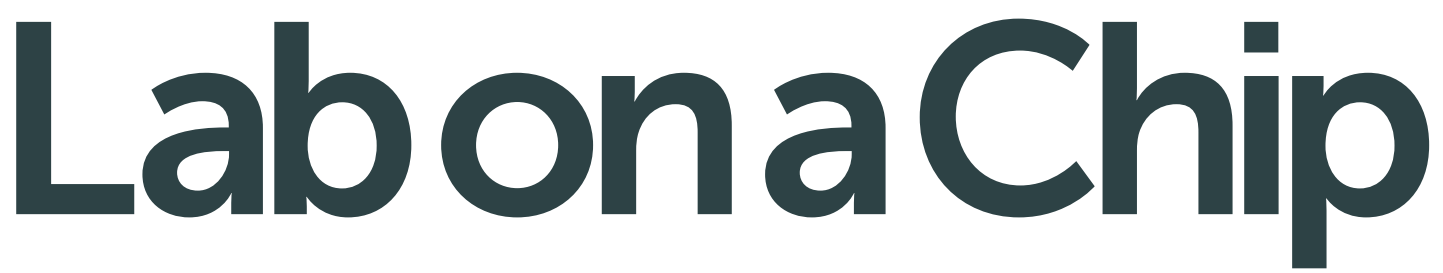

Miniaturisation for chemistry, physics, biology, materials science and bioengineering www.rsc.org/loc

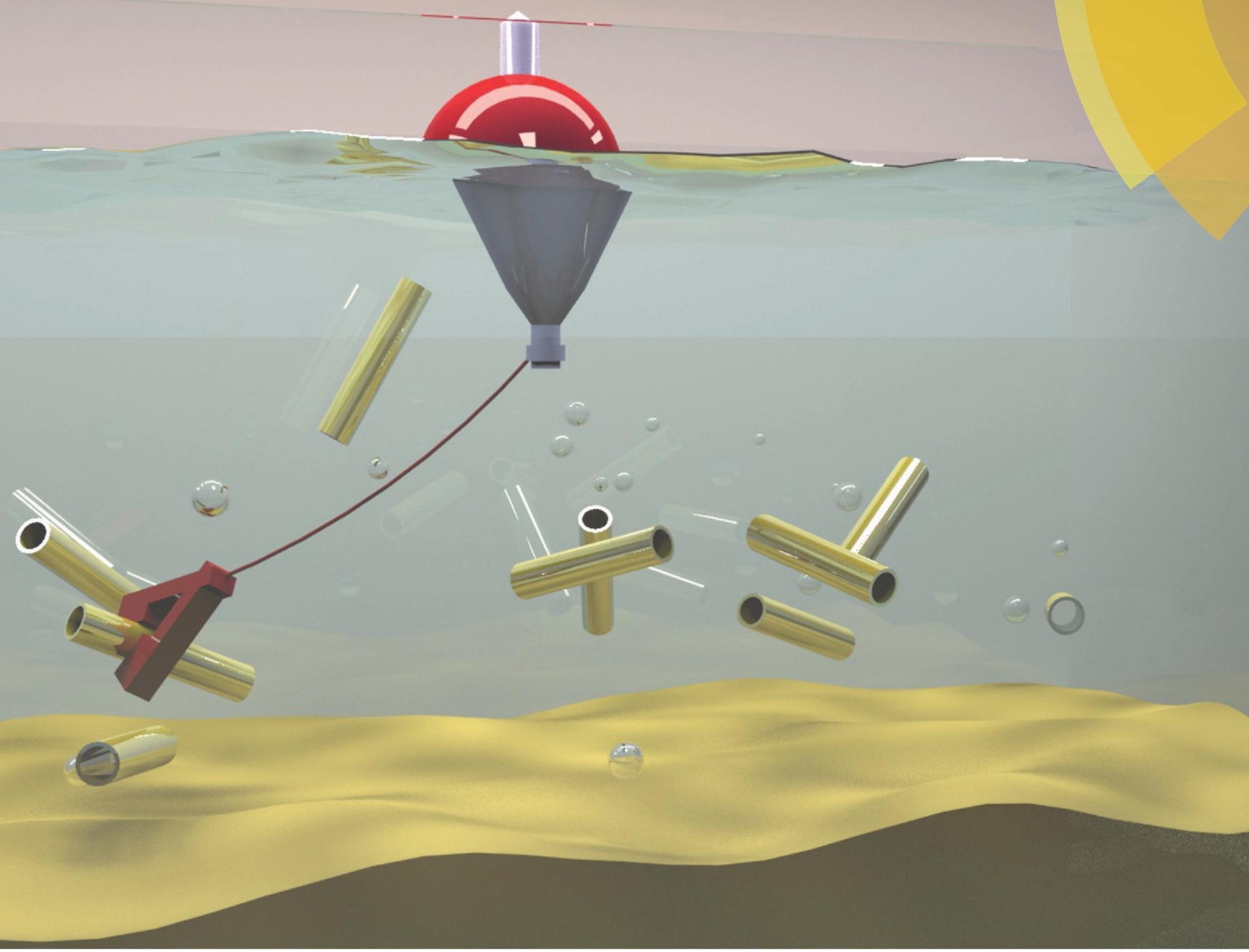

ISSN 1473-0197 


\title{
Trapping self-propelled micromotors with microfabricated chevron and heart-shaped chips $\dagger$
}

Cite this: Lab Chip, 2014, 14, 1515

\author{
Laura Restrepo-Pérez, ${ }^{a}$ Lluís Soler, ${ }^{\text {ab }}$ Cynthia S. Martínez-Cisneros, ${ }^{a}$ \\ Samuel Sánchez ${ }^{* a b}$ and Oliver G. Schmidt ${ }^{\text {ac }}$
}

Received 20th December 2013,

Accepted 10th February 2014

DOI: $10.1039 / c 31$ c51419f

www.rsc.org/loc

We demonstrate that catalytic micromotors can be trapped in microfluidic chips containing chevron and heart-shaped structures. Despite the challenge presented by the reduced size of the traps, microfluidic chips with different trapping geometries can be fabricated via replica moulding. We prove that these microfluidic chips can capture micromotors without the need for any external mechanism to control their motion.

Over years of evolution, nature has created powerful microand nano-machines, such as the kinesin motors, that are able to convert chemical energy into mechanical work for the transport of cargo and the performance of multiple tasks at the cellular and molecular levels. These machines have inspired scientists, who envision the construction of miniature devices able of autonomous and efficient propulsion. ${ }^{1-3}$ However, it was only recently, with the advent of nanotechnology, that the fabrication of self-propelled and powerful artificial micromotors was achieved. Multiple geometries and propulsion mechanisms have been reported in the last decade. Janus spherical particles, ${ }^{4-9}$ bimetallic nanowires, ${ }^{10,11}$ screw-shaped wires $^{12-16}$ and catalytic microjet engines ${ }^{17,18}$ are the most common systems. Among them, the catalytic microjet engines or catalytic tubular micromotors, propelled by the ejection of gas bubbles, have gained great attention due to their high speed and propulsion power. ${ }^{17,19}$ These characteristics make them attractive for multiple biological and environmental ${ }^{20-22}$ applications that require cargo load and transport. For many of these applications, the possibility to confine or trap micromotors is

\footnotetext{
${ }^{a}$ Institute for Integrative Nanosciences, Leibniz Institute for Solid State and Materials Research Dresden, Helmholtzstraße 20, 01069 Dresden, Germany ${ }^{b}$ Max Planck Institute for Intelligent Systems, Heisenbergstr. 3, D-70569 Stuttgart, Germany.E-mail: sanchez@is.mpg.de

${ }^{c}$ Materials Systems for Nanoelectronics, TU Chemnitz, 09107 Chemnitz, Germany $\dagger$ Electronic supplementary information (ESI) available: Supporting videos (S1; S2 and S3). See DOI: 10.1039/c3lc51419f
}

desired as it could allow the isolation and concentration of specific components, such as contaminants or biological entities.

Previous studies have shown the possibility to control the speed and directionality of these micromotors using external mechanisms such as magnetic fields, ${ }^{23,24}$ light, ${ }^{25,26}$ ultrasound $^{27,28}$ or temperature. ${ }^{29,30}$ However, to our knowledge, the trapping of micromotors using patterned structures to confine the space where they swim, without the use of external sources, has not been experimentally reported.

While methods for trapping objects at the macroscale are well established and have been used since ancient times (i.e. for fishing and hunting), trapping self-propelled objects at the microscale becomes challenging due to the strongly reduced size of the traps. In a recent publication, Löwen's group reported a theoretical model in which static chevronshaped structures can be used to trap self-propelled rod-like particles. ${ }^{31}$ Additionally, ratchets of different geometries have been previously proposed to redirect the motion of motile entities such as bacteria and molecular motors. ${ }^{32,33}$

Here, we integrate these two types of structures - chevrons and ratchets - to demonstrate the trapping and confinement of micromotors due to the steric hindrance that these physical boundaries cause on their movement. For this purpose, we developed a series of microfluidic chips containing patterns of different geometries and allowed micromotors to swim freely in these chambers without the influence of any external mechanism.

We first fabricated chips containing chevron-shaped structures and studied the feasibility of using these systems for the trapping of micromotors. We found a direct relation between the angle of the chevron apex and the trapping efficiency of these structures.

We also studied the effect of ratchets on the motion of artificial micromotors and we could observe that the rectifying effect previously reported for biological motile entities is also observed for artificial micromotors. These two structures were 
finally combined to create a microfluidic chip containing a heart-shaped reservoir in which micromotors get trapped over time due to the two previously mentioned mechanisms. We present the use of micropatterned walls for the control of trajectories of self-propelled particles.

To verify the feasibility of trapping micromotors with chevron-shaped structures, we conducted a series of experiments in which micromotors were placed in microfluidic chambers containing chevrons of different angles and without the effect of any external force. Initially, we used microfluidic chips containing single chevrons of $40^{\circ}, 116^{\circ}$ and $140^{\circ}$, following some of the values calculated by Löwen and co-workers. Preliminary experiments verified the influence of the chevron angle on the trapping of micromotors (Fig. 1). For the smallest angle $\left(40^{\circ}\right)$, micromotors remained trapped at the apex of the chevron and a retention time of over $14 \mathrm{~s}$ was found for the presented example in Fig. 1a. Bigger angles $\left(116^{\circ}\right.$ and $\left.140^{\circ}\right)$, on the other hand, allowed micromotors to rapidly escape from the apex and no trapping was observed, as illustrated in Fig. $1 \mathrm{~b}$ and $\mathrm{c}$ (see video $\mathrm{S} 1$ in the ESI†े).

For a more detailed study of the influence of the chevron angle on micromotor trapping, microfluidic chips containing an ensemble of chevrons of different angles were used (Fig. 2a inset).

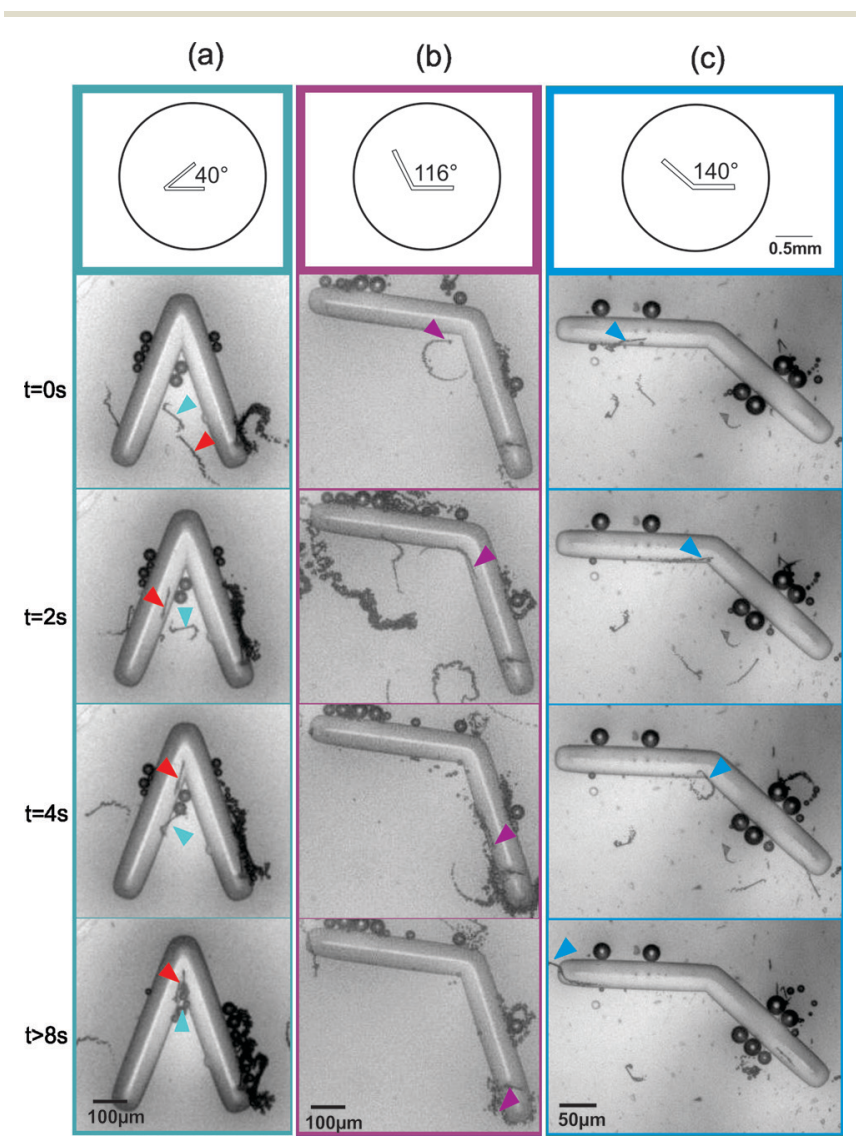

Fig. 1 Time-lapse images of micromotors swimming in microfluidic chips containing chevrons of $40^{\circ}$ (a), $116^{\circ}$ (b) and $140^{\circ}$ (c). The coloured arrows point to the micromotors approaching the chevron apex. Micromotors are confined at the apex of the trap for the $40^{\circ}$ chevron (a), while no confinement is observed for $116^{\circ}$ (b) and $140^{\circ}$ (c).
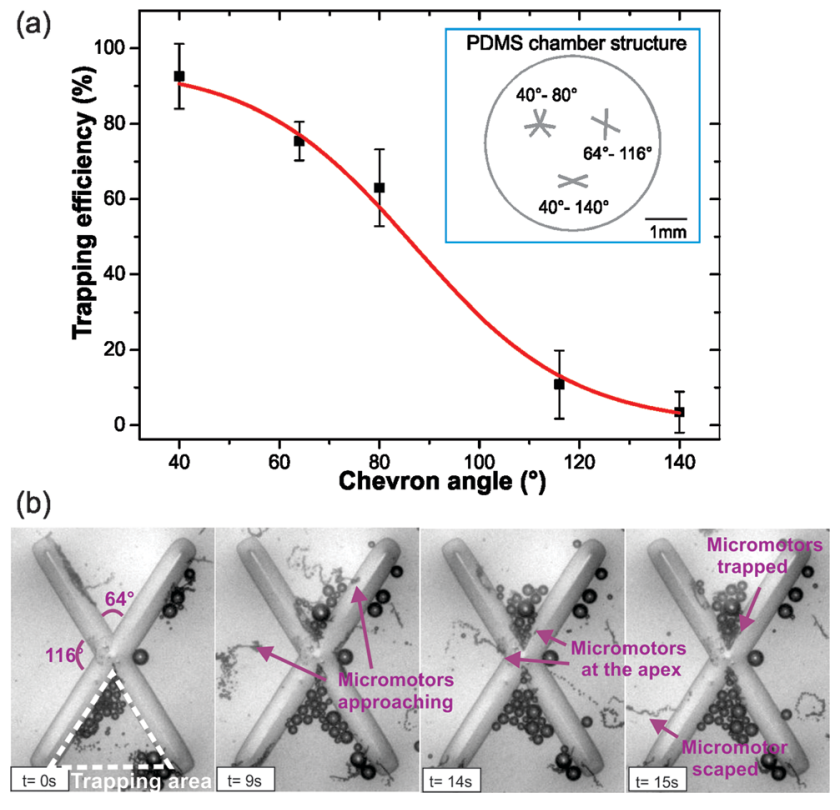

Fig. 2 (a) Trapping efficiency vs. chevron angle. The $x$-axis shows the angle of the chevron structure and the $y$-axis represents the trapping efficiency in percentage. The inset shows a schematic illustration of the PDMS chamber used for the measurements. (b) Time-lapse of micromotors placed in a microfluidic chip containing chevron structures of $64^{\circ}$ and $116^{\circ}$. After $15 \mathrm{~s}$ multiple micromotors are trapped in the $64^{\circ}$ structure while no trapping is observed for the $116^{\circ}$. The chevron area marked with white dashed lines defines the trapping area.

For angles of $40^{\circ}, 64^{\circ}, 80^{\circ}, 116^{\circ}$ and $140^{\circ}$, videos were recorded and a parameter named trapping efficiency was measured. The trapping efficiency was defined as the ratio of the number of micromotors trapped to the number of micromotors that enter the trapping area. The trapping area is defined as the area of the triangle created by the two sides of the trap, as illustrated in Fig. 2b. A micromotor is considered to be trapped when it remains confined in the trapping area for a minimum of 10 seconds and until the end of the video acquisition process ( $c a .50$ seconds). The trapping efficiencies found for these angles are presented in the graph shown in Fig. 2a. As observed with single chevrons, trapping of micromotors is reduced for bigger angles as compared to their smaller counterparts. For our system, the relation between the chevron angle and its trapping efficiency was fitted with a sigmoidal function (coefficient of determination $\left(R^{2}\right)=0.99$ ). Fig. $2 \mathrm{~b}$ shows structures containing $64^{\circ}$ and $116^{\circ}$ angles. In the time-lapse, the violet arrows point at two micromotors approaching the two different angles. Here, we can observe how multiple micromotors are being trapped in the $64^{\circ}$ angle while no trapping is observed for $116^{\circ}$ (see video S2 in the ESI $\dagger$ ).

Opposite to what was observed in the theoretical model proposed by Löwen and coworkers, giant aggregates of micromotors are not observed in our traps. In our case, individual micromotor trapping dominates over collective self-trapping and only small aggregates of a few motors were observed to jam at the chevron apex. This is in accordance with previous studies that report that micromotors swimming under our regular conditions $\left(\mathrm{H}_{2} \mathrm{O}_{2}\right.$ concentration, $2-10 \%$ and $0.01-1 \%$ surfactant $)$ 
(a)

(b)

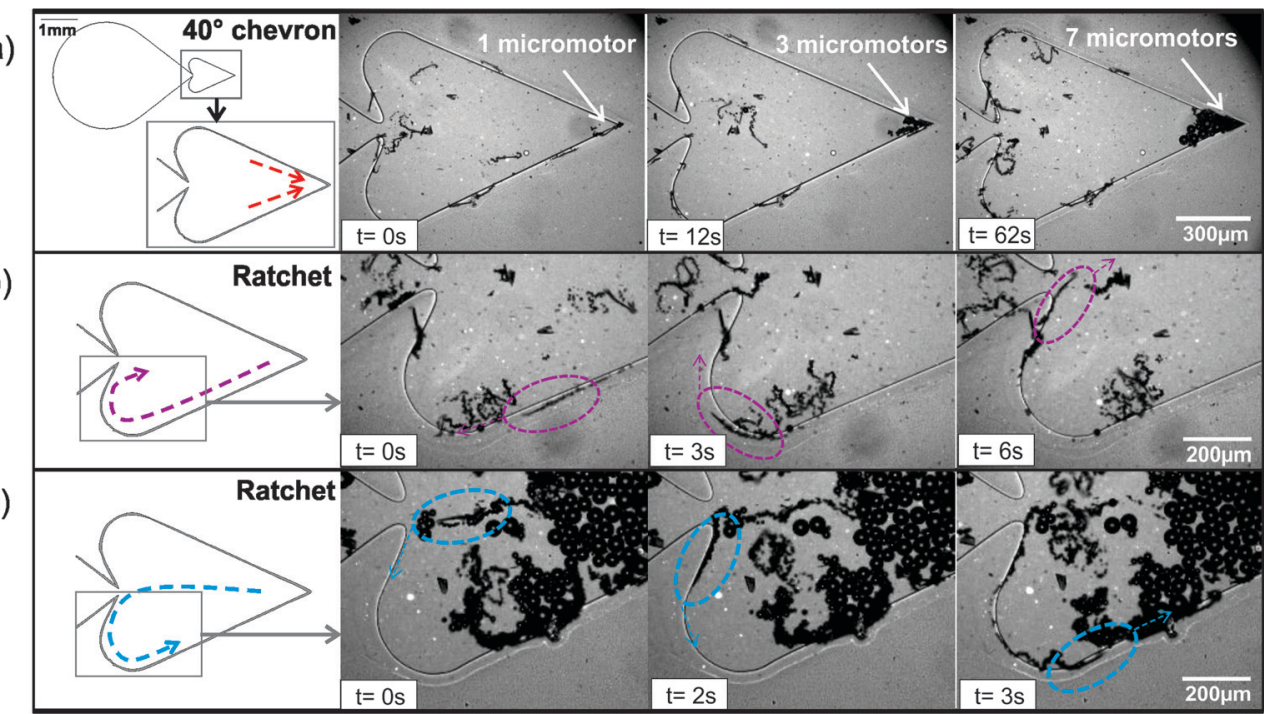

Fig. 3 Trapping mechanisms in the heart-shaped structure. Left panels show the design of the heart-shaped reservoir (a) time lapse images of micromotors being trapped in the $40^{\circ}$ chevron. A total of 7 micromotors are trapped in ca. 1 min (b) Micromotors swimming towards the center and (c) from the center along the walls of the ratchet mechanism are retained in the trapping chamber. The red, pink and blue arrows on the schematics of the left-side figures indicate the trajectories of the micromotors.

do not form swarms. ${ }^{34}$ After investigating the effect of the chevron-shaped structures on micromotor trapping, we fabricated a chip that combined the trapping angle of higher efficiency $\left(40^{\circ}\right)$ with a ratchet structure. In this way, we created a microfluidic chip containing a main reservoir and a heartshaped reservoir (Fig. 3a). The heart-shaped reservoir was intended to concentrate or trap micromotors using two different mechanisms: first, the $40^{\circ}$ angle structure serves as a trapping chevron to avoid the return of the micromotors to the main reservoir and second, the ratchet decreases considerably the amount of motors escaping from the trapping chamber thanks to the rectification of their trajectories towards the right side of the chamber. Fig. 3 shows the trapping mechanism of these two structures (see video S3 in the ESI $\dagger$ ).

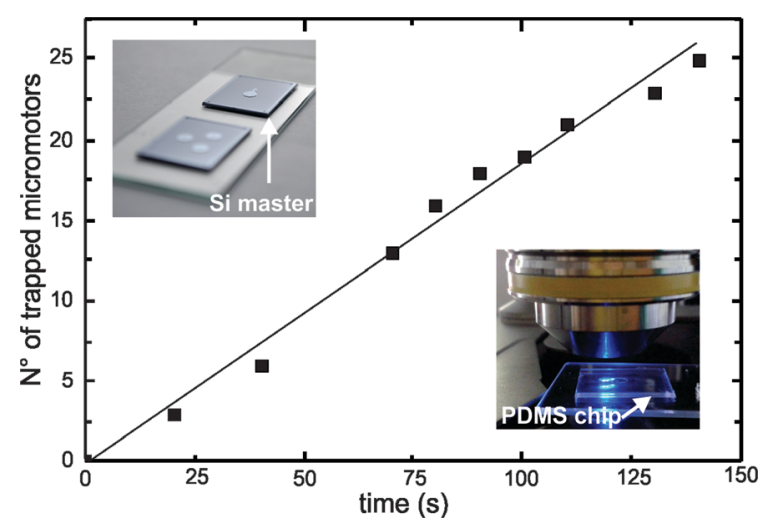

Fig. 4 Micromotors being confined in the heart-shaped reservoir. The graph shows the gradual increase in the number of micromotors present in the chamber over time. The inset on the upper-left corner shows the silicon master structures used to fabricate the PDMS microfluidic chips and the lower-right image shows one of the PDMS microfluidic chips used in our experiments.
To verify the working principle of our system, we quantified the amount of micromotors present in the heart-shaped reservoir over a certain period of time. As shown in Fig. 4, we found a gradual increase of the number of micromotors over time and a maximum of 25 micromotors were trapped in $140 \mathrm{~s}$.

To investigate the effect of the size of the aperture on the heart-shaped reservoir, microfluidic chips were fabricated with three different sizes of the aperture between the main chamber and the heart-shaped chamber: 300,100 and $50 \mu \mathrm{m}$. For each of these cases, we calculated the trapping efficiency of the chamber, which was defined as the ratio of the total number of micromotors that remain trapped in the heart-like chamber to the total number of micromotors that enter the chamber (i.e. motors that remain in the chamber and motors that escape). The results obtained for each case are depicted in Fig. 5. It is

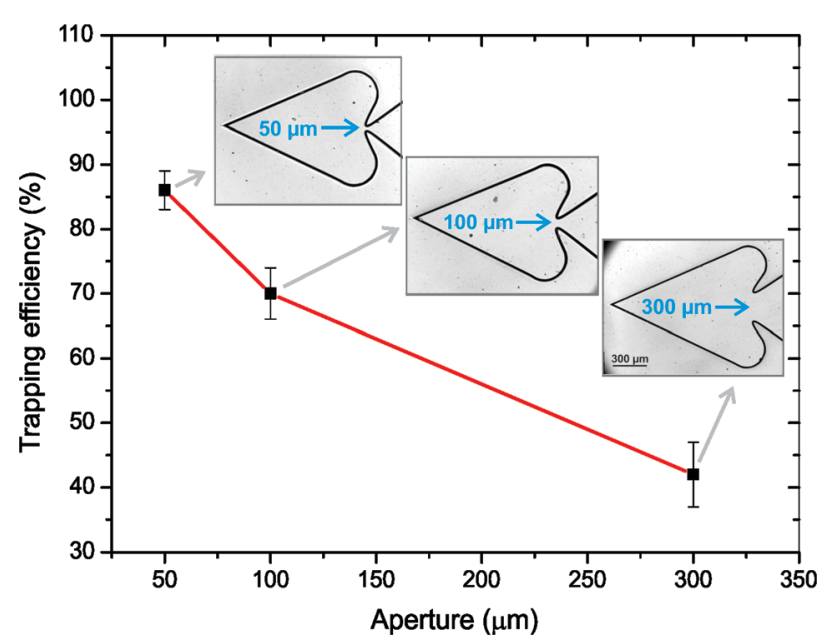

Fig. 5 Trapping efficiency of the heart-shaped chambers with different aperture sizes. The insets show the microchip structures used in each case. 
shown that the higher efficiency is found for the microchip containing the $50 \mu \mathrm{m}$ aperture, which is expected because the space available for micromotors to escape is reduced.

\section{Conclusions}

In this work, we demonstrated the possibility of trapping artificial micromotors. We use microfluidic chips containing chevron and heart-shaped geometries to show how physical boundaries can cause micromotor trapping due to steric restriction of their motion. Our approach not only demonstrates the trapping of micromotors but also eliminates the need for any external mechanism to control their motion, since it merely relies on steric boundaries present in the micromotor environment. This advantage could be especially beneficial for the integration of this mechanism into more complex platforms and would facilitate miniaturization, since no external power source would be required. In the future, these structures could be used in lab-ona-chip systems as a passive mechanism for sample concentration or isolation of cargo. For example, contaminants or biological entities could be confined in specific areas of the system for biosensing or as a pre-concentrating step. Additionally, our system could serve as a filtering device to separate active and inactive micromotors since only active micromotors can swim and get trapped into the heart-shaped reservoir.

\section{Acknowledgements}

The research leading to these results has received funding from the European Research Council under the European Union's Seventh Framework Programme (FP7/2007-2013)/ERC grant agreement no. [311529]. S.S. and L.S. thank DFG (grant SA 2525/1-1) for financial support. The authors also acknowledge Stefan Diez and Veronika Magdanz for active discussion during the performance of the experiments, Ralf Träger for the graphical abstract and Stefan Harazim for performing plasma etching of the silicon masters.

\section{References}

1 W. F. Paxton, K. C. Kistler, C. C. Olmeda, A. Sen, S. K. St Angelo, Y. Cao, T. E. Mallouk, P. E. Lammert and V. H. Crespi, J. Am. Chem. Soc., 2004, 126, 13424-13431.

2 S. Fournier-Bidoz, A. C. Arsenault, I. Manners and G. A. Ozin, Chem. Commun., 2005, 441-443.

3 T. E. Mallouk and A. Sen, Sci. Am., 2009, 300, 72-77.

4 S. Jiang, Q. Chen, M. Tripathy, E. Luijten, K. S. Schweizer and S. Granick, Adv. Mater., 2010, 22, 1060-1071.

5 L. Baraban, D. Makarov, R. Streubel, I. Mönch, D. Grimm, S. Sanchez and O. G. Schmidt, ACS Nano, 2012, 6, 3383-3389.

6 L. Baraban, M. Tasinkevych, M. N. Popescu, S. Sanchez, S. Dietrich and O. G. Schmidt, Soft Matter, 2012, 8, 48.

7 L. F. Valadares, Y.-G. Tao, N. S. Zacharia, V. Kitaev, F. Galembeck, R. Kapral and G. A. Ozin, Small, 2010, 6, 565-572.

8 G. Loget, J. Roche and A. Kuhn, Adv. Mater., 2012, 24, 5111-5116.
9 J. Howse, R. Jones, A. Ryan, T. Gough, R. Vafabakhsh and R. Golestanian, Phys. Rev. Lett., 2007, 99, 048102.

10 J. Wang, ChemPhysChem, 2009, 10, 1748-1755.

11 T. R. Kline, W. F. Paxton, T. E. Mallouk and A. Sen, Angew. Chem., Int. Ed., 2005, 44, 744-746.

12 S. Tottori, L. Zhang, F. Qiu, K. K. Krawczyk, A. Franco-Obregón and B. J. Nelson, Adv. Mater., 2012, 24, 811-816.

13 L. Zhang, J. J. Abbott, L. Dong, B. E. Kratochvil, D. Bell and B. J. Nelson, Appl. Phys. Lett., 2009, 94, 064107.

14 K. E. Peyer, L. Zhang and B. J. Nelson, Nanoscale, 2013, 5, 1259-1272.

15 A. Ghosh and P. Fischer, Nano Lett., 2009, 9, 2243-2245.

16 J. Li, S. Sattayasamitsathit, R. Dong, W. Gao, R. Tam, X. Feng, S. Ai and J. Wang, Nanoscale, DOI: 10.1039/C3NR04760A.

17 A. A. Solovev, Y. Mei, E. Bermúdez Ureña, G. Huang and O. G. Schmidt, Small, 2009, 5, 1688-1692.

18 G. Zhao and M. Pumera, RSC Adv., 2013, 3, 3963.

19 Y. Mei, G. Huang, A. A. Solovev, E. B. Ureña, I. Mönch, F. Ding, T. Reindl, R. K. Y. Fu, P. K. Chu and O. G. Schmidt, Adv. Mater., 2008, 20, 4085-4090.

20 L. Soler, V. Magdanz, V. M. Fomin, S. Sanchez and O. G. Schmidt, ACS Nano, 2013, 7, 9611-9620.

21 M. Guix, J. Orozco, M. García, W. Gao, S. Sattayasamitsathit, A. Merkoçi, A. Escarpa and J. Wang, ACS Nano, 2012, 6, 4445-4451.

22 J. Orozco, G. Cheng, D. Vilela, S. Sattayasamitsathit, R. Vazquez-Duhalt, G. Valdés-Ramírez, O. S. Pak, A. Escarpa, C. Kan and J. Wang, Angew. Chem., Int. Ed., 2013, 52, 13276-13279.

23 A. A. Solovev, S. Sanchez, M. Pumera, Y. F. Mei and O. G. Schmidt, Adv. Funct. Mater., 2010, 20, 2430-2435.

24 I. S. M. Khalil, V. Magdanz, S. Sanchez, O. G. Schmidt and S. Misra, Appl. Phys. Lett., 2013, 103, 172404.

25 A. A. Solovev, E. J. Smith, C. C. Bof' Bufon, S. Sanchez and O. G. Schmidt, Angew. Chem., Int. Ed., 2011, 50, 10875-10878.

26 Y. Hong, M. Diaz, U. M. Córdova-Figueroa and A. Sen, Adv. Funct. Mater., 2010, 20, 1568-1576.

27 V. Garcia-Gradilla, J. Orozco, S. Sattayasamitsathit, F. Soto, F. Kuralay, A. Pourazary, A. Katzenberg, W. Gao, Y. Shen and J. Wang, ACS Nano, 2013, 7, 9232-9240.

28 W. Wang, L. A. Castro, M. Hoyos and T. E. Mallouk, ACS Nano, 2012, 6, 6122-6132.

29 S. Balasubramanian, D. Kagan, K. M. Manesh, P. Calvo-Marzal, G.-U. Flechsig and J. Wang, Small, 2009, 5, 1569-1574.

30 S. Sanchez, A. N. Ananth, V. M. Fomin, M. Viehrig and O. G. Schmidt, J. Am. Chem. Soc., 2011, 133, 14860-14863.

31 A. Kaiser, H. H. Wensink and H. Löwen, Phys. Rev. Lett., 2012, 108, 268307.

32 M. G. L. Van Den Heuvel, C. T. Butcher, R. M. M. Smeets, S. Diez and C. Dekker, Nano Lett., 2005, 5, 1117-1122.

33 S. Elizabeth Hulme, W. R. DiLuzio, S. S. Shevkoplyas, L. Turner, M. Mayer, H. C. Berg and G. M. Whitesides, Lab Chip, 2008, 8, 1888-1895.

34 A. A. Solovev, S. Sanchez and O. G. Schmidt, Nanoscale, 2013, 5, 1284-1293. 